\title{
Immunotherapy on Cancer: Current Progress and Perspective
}

\author{
Weinstein H. Cohn, PhD, DPharm; Hansen Leonardo, Jr, PhD
}

\section{SUMMARY}

Monoclonal antibodies (mAbs) have just turned into an essential piece of the treatment for various cancers. As analysts have found out more about what makes cancer cells different from normal cells, they have created mAbs to exploit these distinctions. Analysts are likewise considering different methods for making monoclonal antibodies more secure and more effective. As said in Immune checkpoint inhibitors to treat cancer, the immune system has checkpoint proteins, (for example, PD-1 and CTLA-4) that help shield it from assaulting other normal cells in the body. Tumor cells here and there exploit these checkpoints to abstain from being assaulted by the immune system. Vaccines are not yet a noteworthy type of treatment for cancer. Scientists have been attempting to create vaccines to battle cancer for quite a long time, however this has ended up being harder than was first idea. Many different types of vaccines are now being studied to treat a variety of cancers e.g. Antigen vaccines, Tumor cell vaccines, Vector-based vaccines and Dendritic cell vaccines. Viruses are a type of germ that can taint and damage cells. Some viruses can be adjusted in the lab with the goal that they taint and damage basically cancer cells; this is a promising better approach to get immune cells called T cells to battle cancer. For this system, T cells are expelled from the patient's blood and hereditarily adjusted in the lab to have particular antigen receptors (called illusory antigen receptors, or CARs) on their surface. Specialists have discovered immune system cells somewhere deep inside a few tumors and have named these cells tumor-infiltrating lymphocytes (TILs).

KEYWORDS Cancer; Therapeutic strategy; Immunity; Prognosis; Outcomes

Sci Insigt. 2017; 2017:e000109. doi:10.15354/si.17.re045

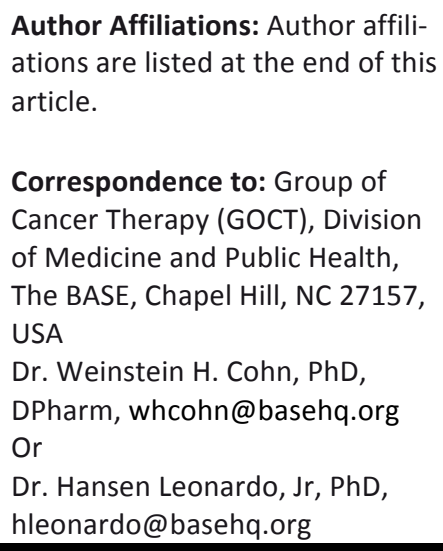

Author Affiliations: Author affiliations are listed at the end of this article.

Correspondence to: Group of Cancer Therapy (GOCT), Division of Medicine and Public Health, The BASE, Chapel Hill, NC 27157, USA

Dr. Weinstein H. Cohn, PhD, DPharm,whcohn@basehq.org Or

Dr. Hansen Leonardo, Jr, PhD, hleonardo@basehq.org 
mmunity therapy on cancer is treatment that uses certain parts of a man's immune system to battle maladies, for example, cancer. This should be possible in a few ways:

- Stimulating your own immune system to work harder or more brilliant to assault tumor cells

- Giving you resistant framework parts, for example, man-made safe framework proteins

Over the most recent couple of decades Immunity therapy on cancer has turned into an imperative piece of treating for cancer. More up to date sorts of safe medicines are presently being considered, and they'll affect how we treat cancer later on.

Immunity therapy on cancer incorporates medications that work in various ways. Some lift the body's immune system in an exceptionally broad manner. Others help prepare the immune system to assault cancer cells particularly.

\section{IMMUNITY THERAPY ON CANCER}

Immune system is a collection of organs, unique cells, and substances that shield you from contaminations and some different maladies. Immune cells and the substances they make venture out through your body to shield it from germs that cause diseases. They additionally help shield you from disease like cancer in some ways.

Your immune system monitors the greater part of the substances typically found in the body. Any new substance that the immune system doesn't recognize raises an alert, making the immune system assault it. For instance, germs contain substances, for example, certain proteins that are not ordinarily found in the human body. The immune system sees these as "outside" and assaults them. The immune system reaction can wreck anything containing the outside substance, for example, germs or cancer cells.

The immune system has a hard time focusing on cancer cells, however. This is on the grounds that cancer begins when cells altered and start to grow out of control. The immune system doesn't generally recognize tumor cells as outside.

Obviously there are restrains on the immune system's capacity to battle cancer all alone, in light of the fact that many individuals with sound immune system still get cancer. At times the immune system doesn't see the growth cells as remote on the grounds that the cells aren't sufficiently diverse from typical cells. Once in a while the immune system recognizes the cancer cells; however the reaction might not be sufficiently solid to wreck the tumor. Cancer cells themselves can likewise emit substances that hold the immune system under tight restraints.

To conquer this, specialists have discovered approaches to enable the insusceptible framework to perceive tumor cells and fortify its reaction so it will wreck them.
The immune system's natural ability to recognize and obliterate unusual cells may keep the improvement of numerous malignancies. Be that as it may, cancer cells are now and then ready to dodge discovery and destruction by the immune system.

Cancer cells may:

- reduce the outflow of tumor antigens on their surface, making it harder for the immune system to distinguish them

- express proteins on their surface that actuate resistant cell inactivation

- induce cells in the encompassing condition (microenvironment) to discharge substances that stifle resistant reactions and advance tumor cell expansion and survival

In some years back, the quickly developing field of disease immunology has created a few new techniques for treating cancer, called immunotherapies that expand the quality of immune reactions against tumors. Immunotherapies either invigorate the exercises of particular segments of the immune system or neutralize signals created by disease cells that stifle immune reactions.

These advances in tumor immunotherapy are the consequence of long haul interests in fundamental research on the immune system - research that continue till date. Extra research is right now under approach to:

- understand why immunotherapy is successful in a few patients however not in other people who have a similar disease

- expand the utilization of immunotherapy to more sorts of malignancy

- increase the adequacy of immunotherapy by joining it with different sorts of disease treatment, for example, directed treatment, chemotherapy, and radiation treatment.

\section{Types of Immunity Therapy on Cancer}

The main types of Immunity therapy on cancer now being used to treat cancer include:

- Monoclonal antibodies: These are man-made renditions of immune system proteins. Antibodies can be extremely valuable in treating malignancy since they can be intended to assault a certain piece of cancer cell.

- Immune checkpoint inhibitors: These medications essentially take the 'brakes' off the immune system, which causes it discover and assault tumor cells.

- Cancer vaccines: Vaccines are substances put into the body to begin a safe reaction against specific infections. We ordinarily consider them being given to sound individuals to help counteract diseases. Be that as it may, a few antibodies can help avert or treat disease.

- Other, non-particular immunotherapies: These medications support the immune system by and 
large, yet this can even enable the immune system to assault tumor cells.

Immunity therapy on cancer drugs are currently used to treat a wide range of malignancy. For more details on Immunity therapy on cancer please keep on reading as the next chapter shared more light on this topic.

\section{* CAR T-Cell Therapy}

The form of ACT that is uttermost along in human clinical trials is CAR T-cell treatment. In this type of ACT, patients' $T$ cells the type of immune cell basically in charge of murdering pathogens are gathered from the blood by means of a method known as apheresis.

These $T$ cells are then hereditarily adjusted in the research laboratory to express a manufactured, or man-made, protein on their surface known as a chimeric antigen receptor, or CAR. The CARs on the T cells are intended to tie to particular proteins on the surface of cancer cells. Being able to tie to the disease cells enables the altered $\mathrm{T}$ cells to assault these cells. This process also spurs the production of other $\mathrm{T}$ cells in the body capable of targeting cancer cells.

After the immune cells are built to express a CAR, they are then developed in the research facility until there are a huge number of them. When they are prepared to be given to patients, patients initially get chemotherapy and different medications that exhaust the assortment of existing T cells. The whole clump of CAR T cells is along these lines injected into the patient in a solitary measurement.

In 2017, FDA affirmed the first CAR T-cell treatment, tisagenlecleucel (Kymriah) for kids and teenagers with intense lymphoblastic leukemia. A second CAR T-cell treatment, for adults with cutting edge lymphomas, might not be far away.

\section{* Immune Checkpoint Modulators}

One immunotherapy approach is to obstruct the capacity of specific proteins, called immune checkpoint proteins, to constrain the quality and length of immune reactions. These proteins typically hold immune reactions under tight check by anticipating excessively extreme reactions that may harm normal cells as well as abnormal cells. Yet, specialists have discovered that cancer can enlist these proteins and utilize them to smother immune reactions.

Hindering the action of immune checkpoint proteins discharges the "brakes" on the immune system, expanding its capacity to damage cancer cells. A few immune checkpoint inhibitors have been endorsed by the Food and Drug Administration (FDA). The first such medication to get endorsement, for the treatment of cutting edge melanoma, hinders the movement of a checkpoint protein known as CTLA4, which is communicated on the surface of enacted immune cells called cytotoxic $\mathrm{T}$ lymphocytes. CTLA4 goes about as a "change" to inactivate these $\mathrm{T}$ cells, accordingly decreasing the quality of immune reactions; ipilimumab ties to CTLA4 and keeps it from sending its inhibitory signs.

Two other FDA-affirmed checkpoint inhibitors, nivolumab (Opdivo) and pembrolizumab (Keytruda), work comparatively, yet they focus on an alternate checkpoint protein on activated T cells known as PD-1. Nivolumab is endorsed to treat a few patients with cutting edge melanoma or advanced lung cancer, and pembrolizumab is affirmed to treat a few patients with cutting edge melanoma.

Scientists have likewise created checkpoint inhibitors that disturb the connection of PD-1 and proteins on the surface of cancer cells known as PD-L1 and PD-L2. Agents that target other checkpoint proteins are likewise being produced, and extra research is gone for understanding why checkpoint inhibitors are effective in a few patients however not in others and distinguishing approaches to grow the utilization of checkpoint inhibitors to other cancer types.

\section{* TCR Therapy}

Another type of ACT, called TCR treatment, is like CAR T-cell treatment. This type of ACT additionally includes building $\mathrm{T}$ cells gathered from patients to express a receptor on their surface-called a T-cell receptor, or TCR.

Not at all like CARs, TCRs are naturally occurring. These receptors enable $\mathrm{T}$ cells to discover antigens from inside cancer cells that have been processed into little bits and transported to and showed on the cell surface.

The procedure for developing TCR cell treatments and administering them to patients is like the procedure for creating CAR T cells.

\section{* Adoptive Cell Transfer: CARs, TCRs, and TILs}

Another type of immunotherapy that is effectively examined and demonstrating enormous guarantee is called adoptive cell transfer (ACT). In a few little clinical trials testing ACT, a few patients with extremely advanced cancers primarily melanoma and blood cancer like leukemia and lymphoma have had their ailment totally destroyed. At times, these treatment reactions have gone on for quite a long time.

ACT utilizes a patient's own immune cells gathered from their blood or specifically from their tumors to treat their cancer. There are three essential ways to deal with ACT that are being tried in human clinical trials.

\section{* Cancer Treatment Vaccines}

The use of cancer treatment vaccines is another way to deal with immunotherapy. These immunizations are normally produced using a patient's own tumor cells or from substances develop by tumor cells. They are intended to treat cancers that have effectively created by reinforcing the body's natural defenses against the disease.

In 2010, FDA approved the first cancer treatment vaccine, sipuleucel-T (Provenge), for use in few men with metastatic prostate cancer. Other therapeutic vaccines are 
being tried in clinical trials to treat a scope of cancer, including cerebrum, breast, and lung cancer.

\section{* TIL Therapy}

The first type of ACT to be tried in people used immune cells gathered from a patient's tumor, called tumorinfiltrating lymphocytes (TILs). TILs are immune cells that normally entered a cancer, and their presence is thought to demonstrate that the immune system is attempting to assault the disease.

In TIL treatment, TILs are gathered from a patients' tumor sample and tested in the lab to distinguish those with the best capacity to discover the patient's tumor cells. Dissimilar to CARs or TCRs, they don't experience any further changes or building. Similarly as with CARs and TCRs, in any case, huge populaces of these TILs are developed in the research laboratory.

The extended TILs are then turned on, or initiated, by treatment with immune system flagging proteins called cytokines. After the patient gets chemotherapy to obliterate their current $\mathrm{T}$ cells, the activated cells are infused into the patient in a single dose.

The thought behind this approach is that the TILs have already demonstrated the capacity to target tumor cells, however there might not have been sufficient of these immune cells in and around the tumor (known as the tumor microenvironment) to destroy it or defeat the signs being discharged by tumor cells that square the immune cells' movement. Presenting huge measures of initiated TILs can beat these obstructions, prompting tumor destruction.

\section{* Therapeutic Antibodies}

Therapeutic antibodies are antibodies made in the lab that are intended to cause the destruction of cancer cells. One class of therapeutic antibodies, called antibody drug conjugates (ADCs), has turned out to be especially successful, with a few ADCs having been affirmed by FDA for the treatment of various cancers.

ADCs are made by synthetically connecting antibodies, or parts of antibodies, to a lethal substance. The immune response segment of the ADC enables it to tie to an objective atom that is communicated on the surface of cancer cells. The harmful substance can be a toxic substance, for example, a bacterial poison; a little particle sedate; or a radioactive compound. Once an ADC ties to a tumor cell, it is taken up by the cell and the dangerous substance damage the cell.

FDA has affirmed a few ADCs for the treatment of patients with tumor, including:

- Ado-trastuzumab emtansine (Kadcyla) for the treatment of a few sorts of bosom disease

- brentuximab vedotin (Adcetris) for Hodgkin lymphoma and a sort of non-Hodgkin T-cell lymphoma
- ibritumomab tiuxetan (Zevalin) for a sort of nonHodgkin B-cell lymphoma

Other therapeutic antibodies don't convey harmful payloads. Some of these antibodies make cancer cells to commit suicide (apoptosis) when they tie to them. In other cases, antibody binding to cancer cells is recognized by certain immune cells or proteins known collectively as "complement," which are produced by immune cells, and these cells and proteins mediate cancer cell death.

One case of this type of therapeutic antibody is rituximab (Rituxan), which focuses on a protein on the surface of B lymphocytes called CD20. Rituximab has turned into a backbone in the treatment of some B-cell lymphomas and B-cell chronic lymphocytic leukemia. At the point when CD20-communicating cells end up noticeably covered with rituximab, the medication slaughters the cells by prompting apoptosis, and in addition by immunizer subordinate cell intervened cytotoxicity and supplement subordinate cytotoxicity.

Other therapies combine non-antibody immune system molecules and cancer-killing agents. For instance, denileukin diftitox (ONTAK), which is affirmed for the treatment of cutaneous T-cell lymphoma, comprises of the cytokine interleukin-2 (IL-2) connected to a toxin developed by the bacterium Corynebacterium diphtheria, which causes diphtheria. Some leukemia and lymphoma cells express receptors for IL-2 on their surface. Denileukin diftitox utilizes its IL-2 bit to focus on these cancer cells and the diphtheria toxin to destroy them.

\section{* Immune System Modulators}

However another sort of immunotherapy utilizes proteins that typically help direct, or regulate, immune system movement to upgrade the body's immune reaction against tumor. These proteins incorporate cytokines and certain growth factors. Two types of cytokines are utilized to treat patients with disease: interleukins and interferons.

Immune-modulating agents may work through various components. One kind of interferon, for instance, improves a patient's Immune reaction to cancer cells by enacting certain white platelets, for example, natural killer cells and dendritic cells. Late advances in understanding how cytokines fortify immune cells could empower the improvement of more viable immunotherapies and blends of these agents.

Chan who is a specialist said that cost is a big problem. "We've got to try to make these treatments available at a lower cost. The United States pays far higher costs for drugs than other countries. We're the only ones who don't take cost into account before approving a drug. We don't negotiate costs with drug companies. Americans are bearing the price of drug research for the entire world," he said.

Immunotherapy is often covered by health insurance, but patients still have to deal with rising out-of-pocket costs. Surgery and other cancer treatments add still more to the financial burden. 
Over the most recent 5 years, immune checkpoint antibodies have turned out to be built up as anticancer specialty for different types of cancer. These counters acting agent drugs, to be specific cytotoxic T-lymphocyte-related antigen, programmed death-1, and programmed death ligand-1 antibodies, have uncovered moderately high reaction rates, the capacity to instigate tough reactions, and clinical adequacy in malignancies not beforehand thought to be helpless to immune-based strategies. In any case, as a result of it's one of a kind components of actuating the host immune system against tumor and in addition expensive cost, immune checkpoint blockade faces novel difficulties in choosing proper patient populaces, observing clinical reactions, and anticipating immune unfavorable occasions. The development of objective criteria for selecting patient populations that are likely to have benefited from these therapies has been vigorously investigated but still remains unclear. In this result, we depict immune checkpoint inhibition particular difficulties with persistent determination and observing, and concentrate on ways to deal with these difficulties. We additionally examine utilizations of the developing field of immunopharmacogenomics for controlling determination and observing for anti-immune checkpoint treatment.

Specialists figure ACT could be the solution to B-cell infections, for example, chronic lymphocytic leukemia. Around 8-in-10 kids who have acute lymphoblastic leukemia (ALL) are dealt with utilizing intensive chemotherapy. In any case, if the cancer returns after treatment (which is quite often does), the patients are left with no further choices. The ACT is another alternative for this group of patients.
Two trials were directed for the CAR T-cells. Every patient was infused with designed $\mathrm{T}$-cells to assault the CD19 antigen, which is on the surface of the B cells.

The first trial, referred to as the CHOP trial, was led in the University of Pennsylvania. Most of the patients reacted decidedly to the treatment. Despite the fact that 3 out of the 17 members were determined to have tumor once more, fourteen remained cancer free.

On the second trial carried out at the National Cancer Institute (NCI) by Daniel W. Lee, nine out of fifteen patients reacted emphatically to the treatment. Amid the trial, two patients got just a little measurement of the CAR Tcells contrasted with alternate patients, nevertheless, those patients likewise responded to the treatment positively.

\section{CONCLUDING REMARKS}

Cancer therapy is thorny problem. Current knowledge using immunity-associated strategies on cancer management is overwhelming and considered as the promise for finding new breakthrough on cancerous malignancies. Even several maneuvers focusing on various immunity facets have been being identified and practiced on cancer therapy; it is not that easy to get a way, no matter how easy or complicated, to tune the protocol in which dosing, timing, interacting, and side effects etc. to precisely target on cancerous tissues. Giving hope on such situation in cancer therapy to being not "wishes more, but lose more".

\section{ARTICLE INFORMATION}

Author Affiliations: Group of Cancer Therapy (GOCT), Division of Medicine and Public Health, The BASE, Chapel Hill, NC 27157, USA.

Author Contributions: Both authors had full access to all of the data in the study and take responsibility for the integrity of the data and the accuracy of the data analysis.

Study concept and design: All authors.

Acquisition, analysis, or interpretation of data: All authors.

Drafting of the manuscript: All authors.
Critical revision of the manuscript for important intellectual content: All authors. Statistical analysis: N/A.

Obtained funding: N/A.

Administrative, technical, or material support: Keiser.

Study supervision: All authors.

Conflict of Interest Disclosures: The author declared no competing interests of this manuscript submitted for publication.

Acknowledgement: N/A.

Funding/Support: N/A.
Role of the Funder/Sponsor: N/A.

How to Cite This Paper: Cohn WH, Leonardo H. Immunotherapy on cancer: Current progress and perspective. Sci Insigt. 2017; 2017:e000109.

Digital Object Identifier (DOI): http://dx.doi.org/10.15354/si.17.re045.

Article Submission Information: Received, September 01 2017; Revised: September 27, 2017; Accepted: October 02, 2017. 
1. CAR T Cells: Engineering Patients' Immune Cells to Treat Their Cancers/ http://www.ccn.com
2. Adopting Bodily Defenses to Cure Cancer/ http://www.wikipedia.com
3. Cancer immunotherapy in children: How does it differ from approaches in adults?/ http://www.cancerhelp.org/.. 